\title{
Heartbeat: improved diagnosis of familial hypercholesterolaemia
}

Familial hypercholesterolaemia $(\mathrm{FH})$ is the most common autosomal dominant genetic condition, affecting about 1 in 250 people, caused by a pathogenic variant in one of several genes involved in lipoprotein cholesterol catabolism. Treatment of elevated serum low-density lipoprotein cholesterol in people with FH substantially reduces the risk of ischaemic heart disease and cardiovascular mortality. Yet, the vast majority of $\mathrm{FH}$ cases are undiagnosed and, thus, untreated. Diagnosis is challenging because patients typically are asymptomatic, may not know their family history, are unaware of the seriousness of the diagnosis and may not even be seeing a physician regularly. In addition, the phenotypic diagnosis requires more than just serum cholesterol levels.

In this issue of Heart, Carvalho and colleagues $^{1}$ demonstrated the feasibility of the FH Case Ascertainment Tool (FAMCAT) for identifying patients likely to have $\mathrm{FH}$ in a cohort of 777128 primary care patients in London. The FAMCAT score is based on systematic screening of routine primary care records for cholesterol measurements, age, triglycerides, family history, diabetes, kidney disease and current use of lipid-lowering drugs (figure 1). The use of FAMCAT to identify patients likely to have $\mathrm{FH}$ could ensure more accurate and rapid diagnosis (and subsequent treatment) for this group of patients at high risk of cardiovascular disease.

A different approach to detection of FH was used by Brett and colleagues ${ }^{2}$ in a cohort of 232, 139 Australian general practice patients. Using a pragmatic twostep approach, they first identified those at higher risk of FH using the TARB-Ex electronic screening tool. Then, in the 1843 $(0.8 \%)$ of patients identified electronically by TARB-Ex, clinical assessment by the physician was used to confirm a high $\mathrm{FH}$ risk the based on the phenotypic Dutch Lipid Clinic Network Criteria score. In a subset of 77 patients with FH, subsequent intensification of lipid-lowering therapy

Division of Cardiology, University of Washington, Seattle, Washington, USA

Correspondence to Professor Catherine M Otto, Division of Cardiology, University of Washington, Seattle, WA 98195, USA; cmotto@uw.edu

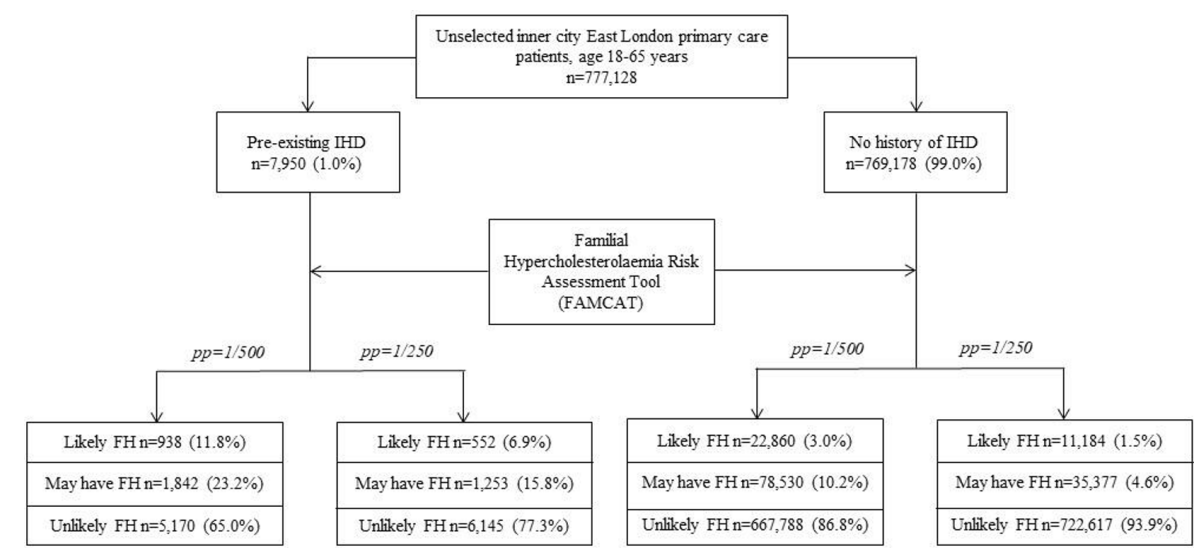

Figure 1 Risk of familial hypercholesterolaemia (FH) in inner East London calculated using FAMCAT algorithm, assuming population prevalence of 1 in 500 and 1 in 250. IHD, ischaemic heart disease; PP, population prevalence.

led to a further reduction in serum cholesterol levels .

In an editorial, Qureshi and Patel $^{3}$ summarise methods using the electronic health record (EHR) for improved diagnosis of FH (figure 2) and point out that the EHR approach often is limited by inadequate or missing data about family history, physical signs and other information. Cholesterol levels, while not diagnostic in isolation, are essential for the diagnosis but may not have been measured in many asymptomatic individuals. They conclude: 'Ultimately, successfully identifying the thousands of people with $\mathrm{FH}$ in the UK and abroad will require a systemwide approach from opportunistic identification at routine health encounters, systematic case finding in primary care, screening people at the time of a premature CVD event to child-parent screening and cascade testing.'
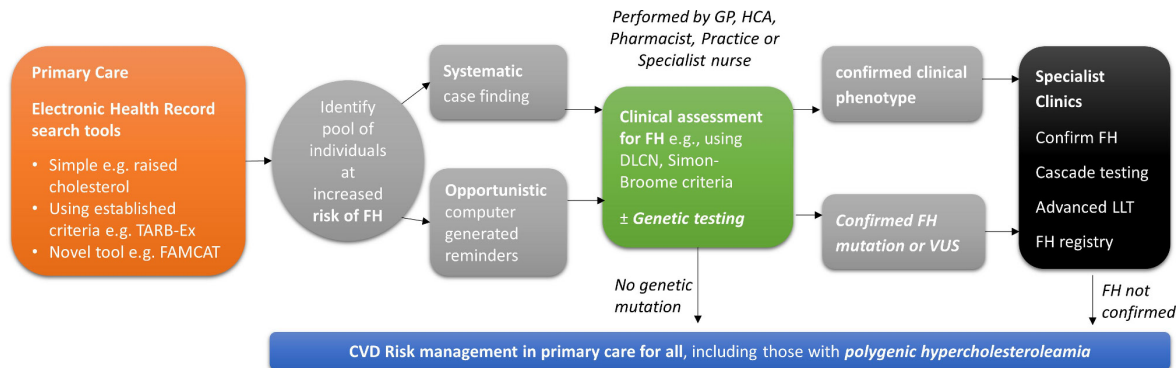

Figure 2 Pathway to identification of FH from primary care. CVD, cardiovascular disease; DLCN, Dutch Lipid Clinic Network; FAMCAT, FH Case Ascertainment Tool; FH, familial hypercholesterolaemia; GP, general practitioner; HCA, healthcare assistant; LLT, lipid-lowering treatment; VUS, variant of unknown significance. 


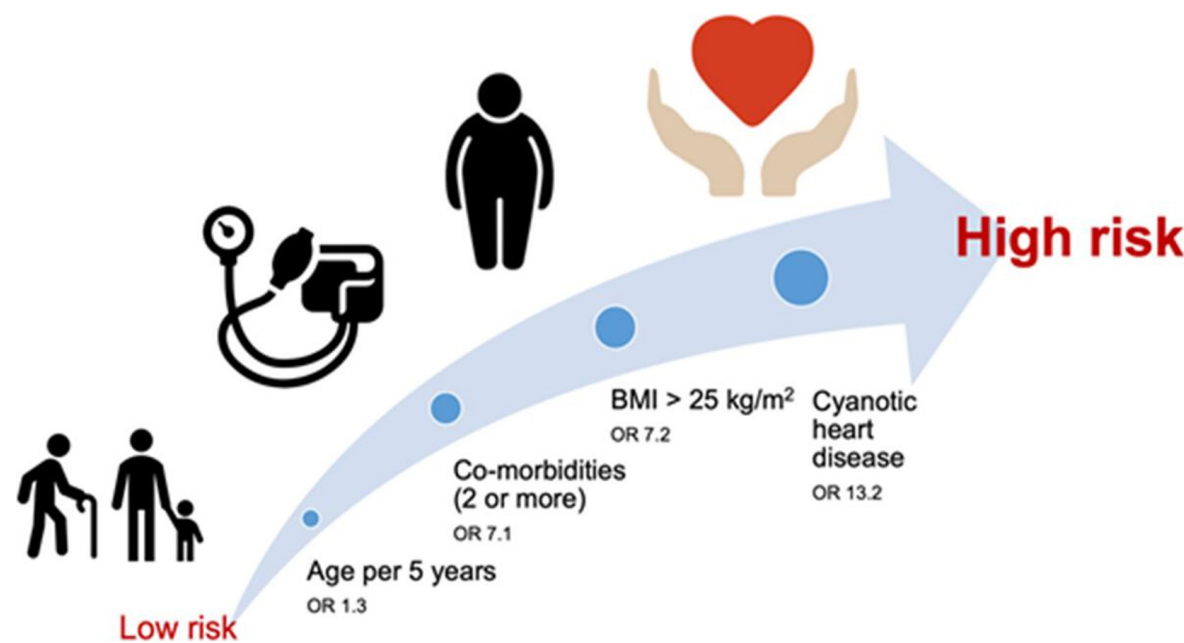

Figure 3 Univariable significant COVID-19 risk factors in patients with adult congenital heart disease and the corresponding ORs. We propose to stratify patients based on age, number of comorbidities, weight and presence of a high-risk cardiac lesion (cyanotic heart disease). BMI, body mass index.

severe infection or death. Rather, patientspecific risk factors similar to those in the non-CHD cohort remain important, while strong CHD-specific risk factors for severe illness or death after COVID-19 infection were cyanotic heart disease and physiological stage. These results help us to tailor patient recommendations but require further confirmation in large international, multicentre studies that are sufficiently powered to answer our remaining questions.'

A meta-analysis by Imazio and colleagues $^{6}$ supports the efficacy of antiinterleukin-1 agents, such as anakinra and rilonacept, for prevention of recurrent episodes of pericarditis in patients with corticosteroid-dependent and colchicine-resistant recurrent pericarditis. Anthony and Collier ${ }^{7}$ remind us that recurrent pericarditis complicates $15 \%-30 \%$ of index cases of pericarditis; the clinical consequences, in addition to pain, can be serious including recurrent effusions, tamponade physiology and constrictive pericarditis; and there is little data on effective therapies (figure 4). ${ }^{8}$ They conclude 'Inhibition of the IL-1 pathway may represent a paradigm shift in the treatment of patients with recurrent pericarditis despite standard therapy. However, larger RCT data are required for further validation of the efficacy and safety of these novel medications in the treatment of recurrent pericarditis.'
The Education in Heart article in this issue provides a quick overview of cardiooncology for the general cardiologist. Cardio-oncology is defined as 'the treatment and prevention of cardiovascular disease in cancer patients both during oncology treatment and afterwards. ${ }^{9}$

A basic understanding of cardiooncology now is considered core knowledge for every cardiologist, given the demographic overlap in the prevalence of cardiovascular disease and cancer, in addition to the potential cardiotoxic effects of cancer treatments. The information and practical advice in this review article are a concise resource for busy practitioners.

Our short Cardiology in Focus article ${ }^{10}$ provides a brief overview of costeffectiveness methodology, with a short list of references for those who wish to dive deeper into this topic.

Funding The authors have not declared a specific grant for this research from any funding agency in the public, commercial or not-for-profit sectors.

\section{Competing interests None declared.}

Patient and public involvement Patients and/or the public were not involved in the design, or conduct, or reporting, or dissemination plans of this research.

Patient consent for publication Not required.

Provenance and peer review Commissioned; internally peer reviewed.

(c) Author(s) (or their employer(s)) 2021. No commercial re-use. See rights and permissions. Published by BMJ.

\section{A Check for updates}

To cite Otto CM. Heart 2021;107:1185-1187.

Heart 2021;107:1185-1187

doi:10.1136/heartjnl-2021-319928
In addition to inflammatory cytokines such as IL-6, promotion and progression of the inflammatory process in pericarditis is due to IL- $1 \alpha$ and IL- $1 \beta$
Autoinflammatory Cycle of RP: Tissue damage caused by IL- $1 \alpha$ and IL-1 $\beta$ in the pericardium stimulates additional $\mathrm{LL}-1 \alpha$ and $\mathrm{IL}-1 \beta$, thereby creating a cyclo of perpotual pericardial inflammation 
ORCID iD

Catherine M Otto http://orcid.org/0000-0002-05279392

\section{REFERENCES}

1 Carvalho C, Williams C, Raisi-Estabragh Z. Application of a risk stratification tool for familial hypercholesterolaemia in primary care: an observational cross-sectional study in an unselected urban population. Heart 2021;107:1220-5.

2 Brett T, Chan DC, Radford J, et al. Improving detection and management of familial hypercholesterolaemia in Australian general practice. Heart 2021;107:1213-9.
3 Qureshi N, Patel RS. Hiding in plain sight: supporting primary care to find familial hypercholesterolaemia and save lives. Heart 2021;107:1190-2.

4 Schwerzmann M, Ruperti-Repilado FJ, Baumgartner H. Clinical outcome of COVID-19 in patients with adult congenital heart disease. Heart 2021;107:1226-32.

5 Yuan S, Oechslin E. Anatomical complexity does not predict outcomes after COVID-19 in adults with congenital heart disease. Heart 2021;107:1193-5.

6 Imazio M, Andreis A, Piroli F, et al. Anti-interleukin 1 agents for the treatment of recurrent pericarditis: a systematic review and meta-analysis. Heart 2021;107:1240-5.
7 Anthony C, Collier P. Anti-Interleukin-1 for recurrent pericarditis; maybe a fix (but prior studies do not really mix). Heart 2021;107:1196-8.

8 Klein AL, Imazio M, Brucato A, et al. RHAPSODY: rationale for and design of a pivotal phase 3 trial to assess efficacy and safety of rilonacept, an interleukin$1 \alpha$ and interleukin- $1 \beta$ trap, in patients with recurrent pericarditis. Am Heart J 2020;228:81-90.

9 Tan L-L, Lyon AR. Cardio-oncology for the general cardiologist. Heart 2021;107:1254-66.

10 Ferro EG, Liu C-L, Kazi DS. Cost-effectiveness and affordability of novel cardiovascular therapies: what physicians need to know. Heart 2021;107:1267-8. 